\title{
POTENCIAL ALELOPÁTICO DE Pyrostegia venusta (Bignoniaceae) SOBRE A GERMINAÇÃO E CRESCIMENTO RADICULAR DE COUVE-DA-MALÁSIA
}

\author{
Regildo Márcio Gonçalves da Silva ${ }^{1}$, Valter Henrique Marinho dos Santos ${ }^{2}$, Gabriel Silva \\ Daneluzzi ${ }^{3}$, Luciana Pereira Silva ${ }^{1}$ \\ ${ }^{1}$ UNESP, Faculdade de Ciências e Letras de Assis, Departamento de Ciências Biológicas, Laboratório de Fitoterápicos \\ (FITOLAB), CEP: 19806-900. Av. Dom Antônio, n²100, Parque Universitário, Assis, São Paulo. regildo@ yahoo.com.br \\ ${ }^{2}$ UNESP/IBB - Distrito de Rubião Júnior - 18618-970 - Botucatu, SP - Brasil. \\ ${ }^{3}$ USP/ESALQ - Av. Pádua Dias, 11 - 13418-900 - Piracicaba, SP - Brasil.
}

\section{RESUMO}

O estudo alelopático investiga os efeitos positivos e negativos que metabólitos secundários de plantas, microrganismos ou fungos exercem sobre o desenvolvimento de indivíduos vizinhos. Pyrostegia venusta (Ker-Gaw) Miers (Bignoniaceae) é uma espécie invasora, utilizada na medicina popular e com metabólitos secundários bem caracterizados. Nesse trabalho foram investigados os efeitos de extrato hidroalcoólico de flores de $P$. venusta sobre a germinação e desenvolvimento do sistema radicular de sementes de Brassica chinensis L. var. parachinensis (Bailey) Sinskaja (couve-da-malásia). O extrato foi preparado adicionando o material vegetal seco e moído (flor) à solução de etanol e água destilada a 70\% na proporção de 1:10 (p/v) e após 24 horas foi filtrado em baixa pressão sob vácuo. Para a realização dos bioensaios, o extrato foi diluídos em $1 \mathrm{~mL}$ de água destilada nas concentrações de 5, $10 \mathrm{ou} 20 \mathrm{mg}$ $\mathrm{mL}^{-1}$. As diferentes concentrações do extrato mostraram alteração nos parâmetros germinativos e no comprimento radicular da planta-teste couve-da-malásia. Os resultados indicam a existência de potencial alelopático em $P$. venusta, uma vez que as diferentes concentrações do extrato causaram alterações metabólicas e morfológicas nas sementes e plântulas de couve-da-malásia e, portanto, a $P$. venusta pode contribuir de forma significativa no estudo das interações químicas entre plantas e na possível produção de um bioherbicida.

Palavras-chave: fitotoxicidade, germinação, bioherbicida, comprimento radicular, couve-damalásia

\section{ABSTRACT}

The allelopathic study investigates the positive and negative effects that secondary metabolites of plants, microorganisms or fungi have on the development of neighboring individuals. Pyrostegia venusta (Ker-Gaw) Miers (Bignoniaceae) is an invasive species, used in the traditional folk medicine and has well characterized secondary metabolites. In this work, it was evaluated the effects of a hydroalcoholic extract of $P$. venusta flowers on Brassica chinensis L. var. parachinensis (Bailey) Sinskaja (tsoi sum) germination and root growth. The extract was prepared by adding the dried and ground plant material (flower) to the solution of ethanol and distilled water to $70 \%$ at a ratio of 1:10 (w / v) and after 24 hours it was filtered at low vacuum pressure. To perform the bioassays, the extract was diluted in $1 \mathrm{~mL}$ of distilled water at concentrations of 5,10 or $20 \mathrm{mg} \mathrm{mL}^{-1}$. The different concentrations of the extract caused changes in the germination parameters and root length of the test plant $B$. chinensis. The results indicate the existence of allelopathic potential in $P$. venusta, since different concentrations of the extract caused morphological and metabolic changes in seeds and seedlings of B. chinensis and, 
therefore, $P$. venusta contributes significantly to the study of chemical interactions between plants and to the possible production of a bioherbicide.

Key words: phytotoxicity, germination, bioherbicide, root length, tsoi sum

\section{INTRODUÇÃO}

O gênero Pyrostegia constituinte da família Bignoniaceae está distribuído em regiões tropicais e subtropicais, particularmente na América do Sul (Judd et al., 1999). A espécie Pyrostegia venusta (Ker Gawl.) Miers, conhecida popularmente como cipó-de-fogo, cipó-de-são-joão e cipóde-lagarto é cultivada pelo aspecto vistoso e pelas reputadas propriedades terapêuticas, sendo utilizada na medicina folclórica tradicional no tratamento de vitiligo. Agronomicamente é considerada invasora, planta que se multiplica rapidamente cobrindo toda a área de ocorrência. É uma espécie com expressiva dispersão na Argentina, Paraguai e em quase todo o sul e sudeste do Brasil, ocorrendo em ambientes higrófito e mesófito, encontrada nas bordas das matas e campos cerrados, também ocorrendo no litoral e beira de estradas (LORENZI, 1991; ACEVEDORODRÍGUEZ, 2005).

Investigações fitoquímicas de $P$. venusta mostraram a presença de diferentes classes de compostos, os quais possuem representantes com potencial alelopático, dentre eles, flavonóides, terpenos, compostos fenólicos, alcalóides, esteróides, ácidos graxos, lactonas insaturadas, taninos, glicosídeos cianogênicos, sesquiterpenos, ácidos fenólicos e outros (MALHEIROS \& PERES, 2001; ALVES \& SANTOS, 2002; KING \& AMBIKA, 2002, VYVYAN, 2002).

A alelopatia é um fenômeno químico-ecológico no qual metabólitos secundários, neste contexto chamados de aleloquímicos, produzidos por uma espécie vegetal são liberados no ambiente interferindo na germinação e/ou desenvolvimento de outras plantas. Num sentido amplo, os efeitos alelopáticos se referem tanto a inibição quanto ao estímulo ao desenvolvimento de outros organismos (RICE, 1984; SANTOS et al., 2003; ALVES et al., 2004).

Vários estudos foram conduzidos nos últimos anos em plantas de cerrado visando identificar propriedades alelopáticas em espécies com potencial para compor sistemas agro-florestais e silvipastoris no Brasil (BORGES et al., 1993; MARASCHIN-SILVA \& AQÜILA, 2006). Além disso, a atividade dos aleloquímicos tem sido usada como alternativa ao uso de herbicidas e inseticidas objetivando o manejo sustentável e ecológico da produção agrícola (WALLER, 1999).

Tendo em vista a capacidade invasora de $P$. venusta e seu perfil fitoquímico, o presente estudo teve como objetivo verificar o potencial alelopático do extrato hidroalcoólico de flores dessa planta por meio de bioensaios de germinação e crescimento radicular de Brassica chinensis (couve-da-malásia).

\section{MATERIAL E MÉTODOS}

As flores de $P$. venusta foram coletadas na região de cerrado no mês de junho de 2009 (localização: 22³2'20''S e $50^{\circ} 22^{\prime} 60^{\prime}$ 'W). A exsicata foi depositada no herbário do Instituto Florestal do Estado de São Paulo (registro: SPSF40207). Após a coleta, o material vegetal foi selecionado e seco em estufa de ar corrente a uma temperatura média de $40^{\circ} \mathrm{C}$ por $24 \mathrm{~h}$, em seguida foi triturado em moinho de facas até a obtenção de um pó.

$\mathrm{O}$ extrato hidroalcoólico foi preparado na proporção de 1:10 (p/v) de pó 
do material vegetal em solução de etanol e água destilada a $70 \%$ por maceração mecânica por $24 \mathrm{~h}$ a temperatura ambiente, após esse período o extrato foi filtrado em baixa pressão sob vácuo (RUTHERFORD \& POWRIE, 1993; HAJHASHEMI et al., 2003; BOLIGON et al., 2009). A extração foi realizada em triplicata. Após a obtenção do extrato, o mesmo foi levado ao evaporador rotativo para retirada total do álcool. Em seguida, os extratos obtidos foram reunidos e concentrados em evaporador rotativo à temperatura média de $60^{\circ} \mathrm{C}$. O extrato aquoso resultante foi liofilizado e o resíduo seco foi utilizado nos bioensaios (AQÜILA, 2000; SADRAEI et al., 2003).

$\mathrm{O}$ pH das diferentes concentrações do extrato hidroalcoólico de flores de $P$. venusta foi determinado diretamente nas soluções de extrato em pHmetro (Tecnopon $^{\circledR}$; modelo MPA210). O potencial osmótico dos extratos foi realizado utilizando-se diluições de polietilenoglicol (PEG-6000) para produzir os potenciais osmóticos de $-0,02$ a $-1,0 \mathrm{MPa}$ de acordo com Villela et al., (1991) e Mazzafera (2003). A medida dos Brix de refração para cada concentração de PEG-6000 e de extrato foi realizada em Refratômetro de ABBE e os valores foram utilizados na obtenção dos potenciais osmóticos conforme descrito por Bakke et al., (2006).

$\mathrm{O}$ bioensaio de germinação foi conduzido em placas de Petri forradas com papel de germinação umedecido com $1 \mathrm{~mL}$ dos extratos diluídos em água destilada nas concentrações de 5,10 ou $20 \mathrm{mg} \mathrm{mL}^{-1}$. Cinqüenta sementes Brassica chinensis L. var. parachinensis (Bailey) Sinskaja (couveda-malásia) foram semeadas por placa, separadas em grupo experimental e controle (água destilada), e incubadas por $96 \mathrm{~h}$ em câmara de germinação tipo BOD sob temperatura de $23 \pm 2{ }^{\circ} \mathrm{C}$ e regime de luz de 24h (ALVES et al., 2004). O desenho experimental foi inteiramente casualizado, com quatro repetições para cada concentração do tratamento e do grupo controle. A germinação foi monitorada a cada $6 \mathrm{~h}$, sendo a protrusão da radícula o critério avaliado para germinação como descrito por Ferreira e Aqüila (2000), Maraschin-Silva e Aqüila (2006). A partir dos resultados os seguintes índices foram calculados: germinabilidade (porcentagem de germinação) [( $\left.\left.\sum \mathrm{ni} / \mathrm{A}\right) .100\right]$, tempo médio de germinação $\left[\mathrm{Tm}=\left(\sum\right.\right.$ ni.ti $) / \sum$ ni $]$, velocidade média de germinação $[\mathrm{Vm}$ $=1 / \mathrm{Tm}]$ e sincronismo de germinação $\left[\mathrm{E}=-\sum\right.$ (fi. $\log 2 . \mathrm{fi})]$, onde $\mathrm{A}=$ número total de sementes colocadas para germinar; $\mathrm{ni}=$ número de sementes que germinam em cada tempo ti; ti= tempo entre o início do experimento e a $i$-ésima hora de observação; fi= freqüência relativa de germinação (LABOURIAU, 1983; SANTANA \& RANAL, 2004; PEREIRA et al., 2009).

As sementes que não germinaram durante o ensaio de germinação foram submetidas ao teste de tetrazólio $(2,3,5-$ trifenil cloreto de tetrazólio a $0,5 \%$ ), por 6 horas, sob temperatura de $30^{\circ} \mathrm{C}$ no escuro, conforme descrito por Delouche et al., (1976) e pelas regras para Análise de Sementes (BRASIL, 2009). Foi determinado sua viabilidade e o número de sementes mortas ou dormentes, para caracterizar o estado metabólico das sementes não germinadas (SOUZA, 1994; ALMEIDA et al., 2002; PINHEIRO \& BORGHETTI, 2003).

No bioensaio de crescimento, 25 plântulas de couve-da-malásia (com aproximadamente $2 \mathrm{~mm}$ de raiz primária) foram dispostas em placas de Petri forradas com papel de germinação umedecidos com 1 $\mathrm{mL}$ dos extratos nas concentrações de 5, 10 ou $20 \mathrm{mg} \mathrm{mL}^{-1}$ ou água destilada. As placas foram colocadas em estufa BOD sob as mesmas condições descritas anteriormente (FERREIRA et al., 2008; MARASCHINSILVA \& AQÜILA, 2006). Foram realizadas medições do comprimento das 
raízes, utilizando um paquímetro digital, após 24 e $48 \mathrm{~h}$ do início do experimento (CARMO et al., 2007).

Foram realizados os testes de normalidade de Shapiro-Wilks e homogeneidade de Levene. Os dados transformados mostraram normalidade e as variâncias foram homogêneas, portanto, os dados foram analisados por meio de testes paramétricos ANOVA e Tukey $(\alpha=0,5)$. Tais testes foram realizados no software SISVAR, de acordo com o proposto por Santana e Ranal (2004) e Pereira et al., (2009).

\section{RESULTADOS E DISCUSSÃO}

Com relação à germinabilidade das sementes de couve-da-malásia, nota-se diferença significativa entre as três concentrações e o controle, mas as concentrações de 10 e $20 \mathrm{mg} \cdot \mathrm{mL}^{-1}$ não diferiram entre si, mais diferiram em comparação ao tratamento com a concentração de $5 \mathrm{mg} \cdot \mathrm{ml}^{-1}$ (Tabela 1). Quanto ao tempo e a velocidade média de germinação, os grupos tratados com as concentrações de 10 e $20 \mathrm{mg} \mathrm{mL}^{-1}$ não apresentaram diferença significativa entre si, mas diferiram do grupo tratado com $5 \mathrm{mg}$ $\mathrm{mL}^{-1}$, sendo que ambos os tratamentos diferiram do controle (Tabela 1). Quanto ao sincronismo da germinação, as três concentrações dos extratos diferiram estatisticamente entre si e também quando comparadas ao controle (Tabela 1).

A caracterização físico-química do extrato hidroalcoólico de $P$. venusta revelou que o $\mathrm{pH}$ do mesmo demonstrou baixa variação de valores e baixa acidez, variando entre 6,35 e 6,67. Os valores de potencial osmótico apresentaram variação entre 0,0043 e -0,0123 MPa (Tabela 2).

Nas primeiras $24 \mathrm{~h}$ de exposição ao extrato, as concentrações de 5 e $20 \mathrm{mg} \mathrm{mL}^{-1}$ não apresentaram diferença significativa entre si, somente com relação a concentração de $10 \mathrm{mg} \mathrm{mL}^{-1}$ e ao controle (Tabela 3 ).

Tais tratamentos inibiram significativamente o crescimento das raízes de couve-da-malásia. Após 48 h de exposição, a redução no crescimento radicular se manteve significativa e ocorreu de maneira dose-dependente (Tabela 3).

Tabela 1. Germinabilidade, tempo, velocidade e sincronismo de germinação de sementes de $B$. chinensis (couve-da-malásia) submetidas a diferentes concentrações de extrato hidroalcoólico de $P$. venusta $\left(5,10\right.$ e $\left.20 \mathrm{mg} \mathrm{mL}^{-1}\right)$.

\begin{tabular}{|c|c|c|c|c|}
\hline $\begin{array}{l}\text { Tratamento } \\
\left(\mathrm{mg} \mathrm{mL}^{-1}\right)\end{array}$ & $\mathrm{G} \pm \mathrm{DP}(\%)$ & $\mathrm{Tm} \pm \mathrm{DP}$ (horas) & $\begin{array}{c}\mathrm{Vm} \pm \mathrm{DP} \\
\left(\text { sementes }^{-1}\right)\end{array}$ & $\mathrm{E} \pm \mathrm{DP}$ (bit) \\
\hline 5 & $90,5 \pm 11,6 b$ & $64,01 \pm 5,79 b$ & $0,020 \pm 0,00 \mathrm{~b}$ & $2,780 \pm 0,43 a$ \\
\hline 10 & $57,0 \pm 29,0 \mathrm{c}$ & $76,84 \pm 7,03 \mathrm{c}$ & $0,013 \pm 0,00 \mathrm{c}$ & $2,313 \pm 0,47 \mathrm{c}$ \\
\hline 20 & $50,0 \pm 22,3 c$ & $75,71 \pm 9,11 \mathrm{c}$ & $0,013 \pm 0,00 \mathrm{c}$ & $2,509 \pm 0,48 b$ \\
\hline Controle Água & $100,0 \pm 0,0 \mathrm{a}$ & $35,13 \pm 1,69 a$ & $0,028 \pm 0,00 \mathrm{a}$ & $1,599 \pm 0,16 \mathrm{~d}$ \\
\hline
\end{tabular}


Tabela 2. pH e potencial osmótico de três concentrações do extrato hidroalcoólico de flores de $P$. venusta

\begin{tabular}{ccc}
\hline Concentração $\left(\mathrm{mg} \mathrm{mL}^{-1}\right)$ & $\mathrm{pH}$ & Potencial Osmótico (MPa) \\
\hline 5 & 6,35 & $-0,0043$ \\
10 & 6,55 & $-0,0097$ \\
20 & 6,67 & $-0,0123$ \\
\hline Controle água & 6,79 & 0 \\
\hline
\end{tabular}

Tabela 3. Comprimento radicular de plântulas de B. chinensis (couve-da-malásia) submetidas a diferentes concentrações de extrato hidroalcoólico de P. venusta $\left(5,10\right.$ e $\left.20 \mathrm{mg} \mathrm{mL}^{-1}\right)$ após 24 e 48 h de exposição.

\begin{tabular}{cll}
\hline Tratamento $\left(\mathrm{mg} \mathrm{mL}^{-1}\right)$ & \multicolumn{2}{c}{$24 \mathrm{~h} 4 \mathrm{~h}$} \\
\cline { 2 - 3 } & $\mathrm{X}(\mathrm{mm}) \pm \mathrm{DP}$ & $\mathrm{X}(\mathrm{mm}) \pm \mathrm{DP}$ \\
\hline 5 & $3,51 \pm 0,18 \mathrm{~b}$ & $3,36 \pm 0,14 \mathrm{~b}$ \\
10 & $3,20 \pm 0,26 \mathrm{c}$ & $3,14 \pm 0,25 \mathrm{c}$ \\
20 & $3,40 \pm 0,43 \mathrm{~b}$ & $2,43 \pm 0,26 \mathrm{~d}$ \\
\hline Controle & $7,80 \pm 0,96 \mathrm{a}$ & $13,19 \pm 3,38 \mathrm{a}$ \\
Água & & \\
\hline
\end{tabular}

Dados apresentados como média (X)士desvio padrão (DP). Médias com letras iguais, na coluna, não diferem entre si pelo Teste de Tukey $(\alpha=0,05)$.

De acordo com Lorenzi (1991), $P$. venusta é considerada uma planta invasora que se multiplica rapidamente cobrindo toda a área de ocorrência. Esta característica peculiar faz com que a mesma desenvolva estratégias necessárias a conquista do ambiente, sendo assim, passível de possuir substâncias aleloquímicas, como confirmado pelos bioensaios aplicados neste estudo.

A resistência ou tolerância aos aleloquímicos é uma característica espécieespecífica, neste sentido, as espécies mais sensíveis como a Lactuca sativa (alface), Brassica chinensis (couve-da-malásia), Lycopersicum esculentum (tomate) e Cucumis sativus (pepino) são consideradas plantas indicadoras de atividade alelopática (FERREIRA \& RANAL, 1999). Para que seja indicada como planta-teste, a espécie deve apresentar germinação rápida, uniforme e um grau de sensibilidade que permita expressar os resultados sob baixas concentrações das substâncias alelopáticas (FERREIRA \& AQÜILA, 2000).

Aqüila et al., (1999) mostraram que os aleloquímicos podem agir de maneira diversa dependendo do ambiente e do estágio do ciclo de vida em que a planta alvo se encontra, visto que ambos refletem diferentes estados fisiológicos. Além disso, os efeitos também podem ser variados quando se considera em qual órgão da planta eles estão atuando. Em estudos realizados por Miró et al., (1998), Jacobi e Ferreira (1991) e Aqüila (2000) se observou um efeito alelopático mais pronunciado sobre o desenvolvimento inicial da planta alvo quando comparado à germinação, já que este último processo utiliza reservas da própria semente. Entretanto, os resultados obtidos no presente estudo revelaram efeitos tanto sobre a germinação quanto sobre o desenvolvimento vegetativo. 
Os testes de pré-emergência demonstraram efeito das concentrações do extrato de $P$. venusta na germinabilidade, tempo médio, velocidade média e sincronismo de germinação (Tabela 1). Esses índices são de grande importância já que expressam o grau de organização das reações químicas que ocorrem nas sementes durante a germinação e precisam ser analisados em conjunto, melhorando, dessa forma, a precisão na quantificação do processo germinativo quando os efeitos de aleloquímicos estão sendo analisados (SANTANA et al., 2006). A importância da inibição da germinação por metabólitos secundários vegetais está relacionada à busca por herbicidas menos nocivos ao meio ambiente (VYVYAN, 2002).

As três concentrações do extrato de $P$. venusta causaram diminuição na germinabilidade, velocidade média e sincronismo de germinação, ou seja, o extrato provocou uma desorganização nas reações químicas que ocorrem durante o processo germinativo, como pode ser visto pela diferença entre os tratamentos e o grupo controle.

A influência na velocidade de germinação tem significado ecológico porque plantas que germinam mais rápido podem se estabelecer no ambiente mais rapidamente, se aproveitando das condições ambientais antes que as competidoras germinem (BORGHETTI \& FERREIRA, 2004). Por outro lado, plantas que germinam mais lentamente, o que ocorreu nos tratamentos, podem apresentar tamanho reduzido e, em conseqüência, podem ser mais susceptíveis a estresses tendo menos chances na competição por recursos (JEFFERSON \& PENNACHIO, 2005).

Com relação ao sincronismo de germinação, a interpretação do índice é: quanto menor seu valor, mais sincronizada será 0 processo germinativo, independentemente do número total de sementes que germinaram (SANTANA \&
RANAL, 2004). A estratégia de sincronizar o processo germinativo, bem como de reduzir o tempo médio de germinação, está relacionada à rápida colonização do ambiente (BORGHETTI \& FERREIRA, 2004). Por outro lado, o atraso e o menor sincronismo de germinação de couve-damalásia (Tabela 1), causado pelas três concentrações do extrato de flor de $P$. venusta, mostram a capacidade desta espécie em se sobressair em ralação a outras, confirmando seu potencial alelopático.

A análise do $\mathrm{pH}$ e do potencial osmótico das diferentes concentrações do extrato de $P$. venusta excluiu uma possível interferência desses fatores no processo de germinação, já que os valores obtidos se mantiveram dentro de padrões aceitáveis no que se considera adequado para germinação e crescimento inicial (AQÜILA, 2000) (Tabela 2). De acordo com Laynez-Garsaball e Mendez-Natera (2006), os valores de $\mathrm{pH}$ entre 6,0 e 7,5 são considerados ideais para a germinação da maioria das espécies vegetais, faixa na qual se enquadra os valores de $\mathrm{pH}$ dos extratos de $P$. venusta, indicando que não houve efeito deste parâmetro sobre a germinação e o desenvolvimento das plântulas.

Gatti et al., (2004) recomendam que o potencial osmótico de extratos envolvendo testes de germinação não ultrapasse valores 0,2 $\mathrm{MPa}$, desta forma, é descartada a possibilidade de interferência deste fator nos resultados, reforçando a idéia de que compostos do extrato apresentaram efeitos tóxicos sobre a germinação de sementes de couve-da-malásia. Quanto ao teste de tetrazólio sobre as sementes não germinadas, provenientes do teste de germinação, todas as que foram tratadas com as diferentes concentrações do extrato apresentaram-se viáveis e/ou dormentes, enquanto que as sementes do grupo controle apresentaram-se mortas. As análises foram realizas de acordo com as regras para Análise de Sementes (BRASIL, 2009). 
Resultados obtidos no desenvolvimento inicial de plântulas, ao final de $48 \mathrm{~h}$ de experimentação, mostram significativa redução no crescimento radicular de plântulas de couve-da-malásia tratadas com as três concentrações dos extratos quando comparadas ao grupo controle (Figura 3). Esses dados mostram que o extrato, além de apresentar características citotóxicas no processo de germinação, apresentou também ação fitotóxica para o desenvolvimento das plântulas.

De acordo com Rice (1984), Aqüila et al., (1999) e Mano (2006), a absorção de íons e água pelas raízes é de grande importância para o crescimento e desenvolvimento da planta, e seus estudos mostram efeito alelopático na absorção radicular. Estudos conduzidos por Balke (1985) mostraram que flavonóides presentes em extratos vegetais tem atividade inibitória sobre ATPases de membrana de raízes, enquanto que compostos fenólicos inibem a absorção de minerais pela mudança na permeabilidade de membranas. Pires et al., (2001), Souza et al., (2005) e Ignanci et al., (2006) mostraram ainda interferência na mitose causa por extratos vegetais.

Em função dessas alterações, a alelopatia é reconhecida como um processo ecológico importante em ecossistemas naturais e manejados, influenciando na sucessão vegetal primária e secundária, na estrutura, composição e dinâmica de comunidades vegetais nativas ou cultivadas (CHOU, 1986; RIZVI et al., 1992; REIGOSA et al., 1999; SCRIVANTI et al., 2003). Neste último caso, os aleloquímicos são vistos como alternativa a agroquímicos sintéticos, objetivando o manejo sustentável e ecológico na produção agrícola.

Muitas substâncias alelopáticas apresentam grande potencial para uso no controle biológico de ervas daninhas (PUTNAM \& DUKE 1974; MACHARIA \& PEFFLEY, 1995; ANAYA, 1999; CHOU,
1998; CHUNG et al., 2001), sendo parcial ou totalmente solúveis em água e ativas em baixas concentrações (VYVYAN, 2002). Nesse sentido, muitos estudos estão sendo realizados na tentativa de diminuir o uso de herbicidas comerciais através da alelopatia com o manejo e o controle das ervas daninhas, por meio da rotação de culturas, sistemas adequados de semeadura entre espécies e entre-safras, além de sistemas agroecológicos (DURIGAN \& ALMEIDA, 1993; BARUAH et al., 1994; WESTON, 1996; CHOU et al., 1998; WU et al., 2000; KHAN et al., 2002; KATO-NOGUCHI, 2003). Rizvi et al., (1999) enfatizaram a importância dos sistemas agroflorestais (uso de associações de espécies arbóreas com a agricultura) para o uso sustentável da terra e o aumento da produtividade agrícola. Nessas associações, o conhecimento das potencialidades alelopáticas das espécies é de essencial importância para o sucesso desse sistema de cultivo. Os mesmos autores listaram aproximadamente 80 espécies consideradas agroflorestais com potencialidades alelopáticas sobre diversas outras.

Em contrapartida ao poder fitotóxico, os efeitos de promoção da germinação e do crescimento vegetal causados por aleloquímicos também são de interesse para o manejo agrícola (VYVYAN, 2002).

\section{CONCLUSÃO}

Os resultados indicam que Pyrostegia venusta apresenta componentes aleloquímicos, caracterizando assim seu potencial alelopático sobre a germinação e o desenvolvimento de outras plantas. Tais resultados contribuem para maior compreensão das interações químicas entre vegetais, com possibilidade de desenvolvimento de um herbicida natural para controle de plantas invasoras em áreas de cultivo, minimizando a utilização de 
agroquímicos, conseqüentemente, reduzindo os custos da produção, além de diminuir os riscos de contaminação ambiental. A conclusão definitiva de que esta espécie é alelopática em condições naturais está associada a uma investigação mais ampla, que inclui outras abordagens experimentais, principalmente testes a campo.

\section{AGRADECIMENTOS}

Os autores são gratos à Fundação de Amparo a Pesquisa do Estado de São Paulo (FAPESP - Processo $\mathrm{n}^{\circ}$ 2008/50689-7 e 2008/50688-0) pelo apoio financeiro para realização do trabalho.

\section{REFERÊNCIAS BIBLIOGRÁFICAS}

ACEVEDO-RODRIGUEZ, P. 2005. Vines and climbing plants of Puerto Rico and the Virgin Islands. Contributions from the United States National Herbarium, v. 51, p. $1-483$.

ALMEIDA, F.A.C.; MORAIS, A.M.; CARVALHO, J.M.F.C.; GOUVEIA, J.P.G. 2002. Crioconservação de sementes de mamona das variedades nordestina e pernambucana. Revista Brasileira de Engenharia Agrícola e Ambiental, v. 6, n. 2, p. 295-302.

ALVES, M. da C. S.; MEDEIROS FILHO, S.; INNECCO, R.; TORRES, S.B. 2004. Alelopatia de extratos voláteis na germinação de sementes e no comprimento da raiz de alface. Pesquisa Agropecuária Brasileira, v. 39, n. 11, p. 1083-1086.

ALVES, S.M.; SANTOS, L.S. 2002. Natureza química dos agentes alelopáticos. In: SOUZA FILHO, A.P.S.; ALVES, S.M. Alelopatia princípios básicos e aspectos gerais. Belém: Embrapa Amazônia Oriental. 260 p..
ANAYA, A.L. 1999. Allelopathy as a tool in the management of biotic resources in agroecosystems. Critical Reviews in Plant Science, v. 18, n. 6, p. 697-739.

AQÜILA, M.E.A. 2000. Efeito alelopático de Ilex paraguariensis A. St.-Hil. na germinação e crescimento inicial de Lactuca sativa L. Iheringia - Série Botânica, v. 53, p. 51-66.

AQÜILA, M.E.A., SILVA, L. P., LAUFER, M. P. 1999. Screening bioassay to detect allelopathy in a natural Brazilian woodland: evaluation of Eugenia uniflora (Myrtaceae). In: World Congress on Allelopathy, critical analysis and future prospects. Thunder Bay. Book of Abstracts. Thunder Bay: Int. Allelop, $52 \mathrm{p}$.

BALKE, N.E. 1985. Effects of allelochemicals on mineral uptake and associated physiological process. In: THOMPSON, A.C. (Ed.). The Chemistry of Allelopathy. Washington: Am. Chem. Soc., p. 161-178.

BARUAH, N.C.; SARMA, J.C.; SARMA, S.; SHARMA, R.P. 1994. Seed germination and growth inhibitory cadinenes from Eupatorium adenophorum Spreng. Journal of Chemical Ecology, v. 20, n. 8, p. 18851892.

BOLIGON, A.A.; PEREIRA, R.P.; FELTRIN, A.C., MACHADO, M.M.; JANOVIK, V.; ROCHA, J.B.T.; ATHAYDE, M.L. 2009. Antioxidant activities of flavonol derivatives from the leaves and stem bark of Scutia buxifolia Reiss. Bioresource Technology, v. 100, n. 24, p. 6592-6598. 
BORGES, E.E.L.; LOPES, E.S.; SILVA, G.F. 1993. Avaliação de substâncias alelopáticas em vegetação de uma floresta secundária. 1- Árvores. Revista Árvore, v. 17, n. 1, p. 69-84.

BRASIL. 2009. Ministry of Agriculture and Agrarian Reform. National Department of Plant Production. Regras para análise de sementes. Brasília, Brazil.

CARMO, F.M.S.; BORGES, E.E.L.; TAKAKI, M. 2007. Alelopatia de extratos aquosos de canela-sassafrás (Ocotea odorifera (Vell.) Rohwer). Acta Botanica Brasilica, v. 21, n. 3, p. 697705 .

CHOU, C.H.; KUO, Y.L. 1986. Allelopathic research of subtropical vegetation in Taiwan. III. Alelopathic exclusion of understory by Leucaena leucocephala (Lam.). Journal of Chemical Ecology, v. 12, p. 1431-1448.

CHOU, C.H.; FU, C.Y.; LI, S.Y.; WANG, Y.F. 1998. Allelopathic potential of Acacia confusa and related species in Taiwan. Journal of Chemical Ecology, v. 24, n. 12, p. 2131-2150.

CHUNG, I.M.; AHN, J.K.; YUN, S.J. 2001. Assessment of allelopathic potential of barnyard grass (Echinochloa crus-galli) on rice (Oriza sativa L.) cultivars. Crop Protection, v. 20, n. 10, p. 921-928.

DELOUCHE, J.C.; STILL, T.W.; RASPET, M.; LIENHARD, M. 1976. The tetrazolium test for seed viability, Brasília, 103 p.

DURIGAN, J.C.; ALMEIDA, F.L.S. 1993. Noções sobre a alelopatia. FUNEP, Jaboticabal.
FERREIRA, A.G.; AQÜILA, M.E.A. 2000. Alelopatia: uma área emergente da ecofisiologia. Revista Brasileira de Fisiologia Vegetal, v. 12, p. 175-204.

FERREIRA, A.G.; BORGHETTI, F. 2004. Germinação: do básico ao aplicado. Artmed, Porto Alegre, RS, 323 p.

FERREIRA, N.R.; MEDEIROS, R.B.; SOARES, G.L. 2008. Potencial alelopático do capim-annoni-2 (Eragrostis plana Nees) na germinação de sementes de gramíneas perenes estivais. Revista Brasileira de Sementes, v. 30, n. 2, p. 43- 50.

FERREIRA, W.R.; RANAL, M.A. Germinação de sementes e crescimento de plântulas de Brassica chinensis L. var. parachinensis (Bailey) Sinskaja (Couveda-malásia). Pesquisa Agropecuária Brasileira, Brasília, v. 34, n. 3, p. 353361, 1999.

GATTI, A.B.; PEREZ, S.C.J.G. de A.; LIMA, M.I.S. 2004. Atividade alelopática de extratos aquosos de Aristolochia esperanzae O. Kuntze na germinação e no crescimento de Lactuca sativa L. e Raphanus sativus L. Acta Botanica Brasilica, v. 18, n. 3, p. 459472.

HAJHASHEMI, V.; GHANNADI, A.; SHARIF, B. 2003. Anti-inflammatory and analgesic properties of the leaf extracts and essential oil of Lavandula angustifolia Mill. Journal of Ethnopharmacology, v. 89, n. 1, p. 6771.

JACOBI, U.S.; FERREIRA, A.G. 1991. Efeitos alelopáticos de Mimosa bimucronata (Dc) Ok. sobre espécies cultivadas. Pesquisa Agropecuária Brasileira, v. 26, n. 7, p. 935-943. 
JEFFERSON, L.V.; PENNACHIO, M. 2005. Allelopathic effects of foliage extracts from four Chenopidiaceae species on seed germination. Journal of Arid Environments, v. 55, p. 275-285.

KATO-NOGUCHI, H. 2003. Assessment of allelopathic potential of shoot powder of lemon balm. Scientia Horticulturae, v. 97, n. 3-4, p. 419-423.

KHAN, Z.R.; HASSANALI, A.; OVERHOLT, W.; KHAMIS, T.; HOOPER, A.M.; PICKETT, J.A.; WADHAMS, L.J.; WOODCOCK, C.M. 2002. Control of witchweed Striga hermonthica by intercropping with Desmodium spp., and the mechanism defined as allelopathic. Journal of Chemical Ecology, v. 28, n. 9, p. 18711885.

KING， S.R.; AMBIKA， R. 2002. Allelopathic plants. 5. Chromolaena odorata (L.). Allelopathy Journal, v. 9, n. 1, p. 35-41.

LABOURIAU, L.F.G. 1983. A germinação das sementes. Departamento de Assuntos Científicos e Tecnológicos da Secretaria Geral da Organização dos Estados Americano, Washington, 174 p.

LAYNEZ-GARSABALL, J.A.; MENDEZNATERA, J.R. 2006. Efectos de extractos acuosos del follaje del corocillo (Cyperus rotundus L.) sobre la germinación de semillas y el crecimiento de plântulas de ajonjolí (Sesamum indicum L.) CV. Arapatol S- 15. Idesia, v. 24, n.2, p. 61-75.

LORENZI, H. 1991. Plantas daninhas do Brasil - terrestres, aquáticas, parasitas, tóxicas e medicinais, 2. ed., Plantarum, Nova Odessa. p. 21.
MACHARIA, C.; PEFFLEY, E.B. 1995. Suppression of Amaranthus spinosus and Kochia scoparia: evidence of competition of allelopathy in Allium fistulosum. Crop Protection, v. 14, n. 2, p.155-158.

MALHEIROS, A.; PERES, M.T.L.P. 2001. Alelopatia: Interações químicas entre espécies. In: YNES, R.A.; CALIXTO, J.B. Plantas medicinais sob a ótica da química medicinal moderna. Chapecó: Argos. p. 503-523.

MARASCHIN-SILVA, F.; AQÜILA, M.E.A. 2006. Contribuição ao estudo do potencial alelopático de espécies nativas. Revista Árvore, v. 30, n. 4, p. 547-555.

MAZZAFERA, P. 2003. Efeito alelopático do extrato alcoólico do cravo-da-índia e eugenol. Revista Brasileira de Botânica, v. 26, n. 2, p. 231-238.

MIRÓ, C.P., FERREIRA, A.G., AQÜILA, M.E.A., 1998. Alelopatia de frutos de erva-mate (Ilex paraguariensis) no desenvolvimento do milho. Pesquisa Agropecuária Brasileira, v. 33, n. 8, p. 1261- 1270.

PEREIRA, R.S.; SANTANA, D.G.; RANAL, M.A. 2009. Emergência de plântulas oriundas de sementes recém colhidas e armazenadas de Copaifera langsdorffii Desf. (Caesalpinioideae), triângulo mineiro, Brasil. Revista Árvore, v. 33, n. 4, p. 643-652.

PINHEIRO, F.; BORGHETTI, F. 2003. Light and temperature requirements for germination of seeds of Aechmea nudicaulis (L.) Griesebach and Streptocalyx floribundus (Martius ex Schultes F.) Mez (Bromeliaceae). Acta Botanica Brasilica, v. 17, n. 1, p. 27-35. 
PIRES, N.M.; SOUZA, I.R.P.; PRATES, H.T.; FARIA, T.C.L.; PEREIRA FILHO I.A.; MAGALHÃES P.C. 2001. Efeito do extrato aquoso de leucena sobre o desenvolvimento, índice mitótico e atividade da peroxidase em plântulas de milho. Revista Brasileira de Fisiologia Vegetal, v. 13, n. 1, p. 55-65.

PUTNAM, A.R.; DUKE, W.B. 1974. Biological suppression of weeds evidence for allelopathy in accessions of cucumber. Science, v. 185, p. 370-372.

REIGOSA, M.J.; SÁNCHEZ-MOREIRAS, A.; GONZÁLEZ, L. 1999. Ecophysiological approach in allelopathy. Critical Reviews in Plant Science, v. 18, n. 5, p. 577-608.

RICE, E.L. 1984. Allelopathy, $2^{\text {nd }}$ ed. Academic Press, Orlando, USA, p. 6768.

RIZVI, S.G.H.; RIZVI, V. 1992. Allelopathy: basic and applied aspects. Chapman and Hall, London, 480 p.

RIZVI, S.J.H.; TAHIR, M.; RIZVI, V.; KOHLI, R.K.; ANSARI, A. 1999. Allelopathy interactions in agroflorestry systems. Critical Reviews in Plant Science, v.18, n. 6, p. 773-796.

RUTHERFORD, M.C.; POWRIE, L.W. 1993. Allelochemic control of biomass allocation in interacting shrub species. Journal of Chemical Ecology, v. 19, n. 5, p. 893-906.

SADRAEI, H.; GHANNADI, A.; MALEKSHAHI, K. 2003. Relaxant effect of essential oil of Melissa officinalis and citral on rat ileum contractions. Fitoterapia, v. 74, n. 5, p. 445-452.
SANTANA, D.G.; RANAL, M.A. 2004. Análise da germinação: Um enfoque estatístico. UNB, Brasília, 247 p.

SANTANA, D.G.; RANAL M.A.; MUSTAFA, P.C.V.; SILVA, R.M.G. 2006. Germination measurements to evaluate allelopathic interactions. Allelopathy Journal, v. 17, n. 1, p. 4352.

SCRIVANTI, L.R.; ZUNINO, M.P.; ZYGADLO, J.A. 2003. Tagetes minuta and Schinus areia essential oils as allelopathic agents. Biochemical Systematics and Ecology, v. 31, n. 6, p. 563-572.

SOUZA, S.A.M.; STEIN, V.C.; CATTELAN, L.V.; BOBROWSKI, V.L.; ROCHA, B.H.G. 2005. Utilização de sementes de alface e de rúcula como ensaios biológicos para avaliação do efeito citotóxico e alelopático de extratos aquosos de plantas medicinais. Revista de Biologia e Ciências da Terra, v. 5, p. 3-9.

VILLELA, F.A.; DONI FILHO, L.; SEQUEIRA, E.L. 1991. Table of osmotic potential as a function of polyethilene glycol 6000 concentration and temperature. Pesquisa Agropecuária Brasileira, v. 26, p. 1957-1968.

VYVYAN, J.R. 2002. Allelochemicals as leads for new herbicides and agrochemicals, Tetrahedron, v. 58, n. 9, p. 1631-1646.

WALLER, G.R. 1999. Introduction. In: MACIAS, F.A.; GALINDO, J.C.G; MOLINILLO, J.M.G.; CUTLER, H.G. (eds.). Recent advances in allelopathy. 
Cádiz, Servicio de Publicaciones, Univiversidad de Cádiz, v.1.

WESTON, L.A. 1996. Utilization of allelopathy for weed management in agroecosystems: Allelopathy in cropping systems. Agronomy Journal, v. 88, n. 6, p. $860-866$.

WU, H.; HAIG, T.; PRATLEY, J.; LEMERLE, D.; AN, M. 2000. Distribuition and exudation of allelochemicals in wheat Triticum aestivum. Journal of Chemical Ecology, v. 26, n. 9, p. 2141-2154. 